\title{
On the Design of NFT-Based Communication Systems With Lumped Amplification
}

\author{
Morteza Kamalian, Jaroslaw E. Prilepsky, Son T. Le, and Sergei K. Turitsyn, Fellow, OSA
}

\begin{abstract}
Nonlinear Fourier transform (NFT) based transmission technique relies on the integrability of the nonlinear Schrödinger equation (NLSE). However, the lossless NLSE is not directly applicable for the description of light evolution in fibre links with lumped amplification such as Erbium-doped fibre amplifier (EDFA) because of the nonuniform loss and gain evolution. In this case, the path-averaged model is usually applied as an approximation of the true NLSE model including the fibre loss. However, the inaccuracy of the lossless path-average model, even though being small, can also result in a notable performance degradation in NFT-based transmission systems. In this paper, we extend the theoretical approach, which was first proposed for solitons in EDFA systems, to the case of NFT-based systems to constructively diminish the aforementioned performance penalty. Based on the quantitative analysis of distortions due to the use of pathaverage model, we optimise the signal launch and detection points to minimise the models mismatch. Without loss of generality, we demonstrate how this approach works for the NFT systems that use continuous NFT spectrum modulation (vanishing signals) and NFT main spectrum modulation (periodic signals). Through numerical modelling, we quantify the corresponding improvements in system performance.
\end{abstract}

Index Terms-Fibre-optic communication, nonlinear Fourier transform (NFT), nonlinear inverse synthesis, periodic nonlinear Fourier transform.

\section{INTRODUCTION}

A PPLICATION of NFT [1] for fibre-optic communication, dating back to the celebrated work of Hasegawa and Nyu [2], has attracted a great deal of interest in recent years. The NFT capability to effectively linearise the channel thus removing (or, in reality, suppressing) the nonlinear cross-talk, and new fast numerical methods to perform the NFT operations, have made the NFT a promising approach in overcoming the

Manuscript received May 29, 2017; revised October 5, 2017 and November 1, 2017; accepted November 10, 2017. Date of publication November 16, 2017; date of current version December 8, 2017. This work was supported in part by the U.K. EPSRC Programme under Grant UNLOC EP/J017582/1 and in part by the Russian Science Foundation under Grant 17-72-30006 (S.K.T.). (Corresponding author: Kamalian Kopae.)

M. Kamalian and J. E. Prilepsky are with the Aston Institute of Photonic Technologies, Aston University, Birmingham B4 7ET, U.K. (e-mail: kamaliam@ aston.ac.uk; y.prylepskiy1@aston.ac.uk).

S. T. Le is with Nokia Bell-Labs, Stuttgart 70435, Germany (e-mail: lethaison. tti@gmail.com).

S. K. Turitsyn is with the Aston Institute of Photonic Technologies, Aston University, Birmingham B4 7ET, U.K., and also with the Aston-Novosibirsk International Center for Photonics, Novosibirsk State University, Novosibirsk 630090, Russia (e-mail: s.k.turitsyn@aston.ac.uk).

Original data for this work is available through Aston Research Explorer (http://doi.org/10.17036/researchdata.aston.ac.uk.00000303).

Color versions of one or more of the figures in this paper are available online at http://ieeexplore.ieee.org.

Digital Object Identifier 10.1109/JLT.2017.2775105 nonlinearity-induced limits on the achievable data rate in fibre optic communications [3]-[16]. The basic concept of NFT is the possibility to decompose the solution of the NLSE (the master model describing the light evolution in the fibre), into noninteracting "nonlinear modes" evolving inside the NFT domain in a simple linear manner. This property makes the parameters of these modes to be promising candidates to carry the data along the fibre, the idea which lies behind the most of the NFTbased techniques [4]. However the linear unperturbed evolution of NFT modes along the line takes place only in the case of perfect (so-called integrable) NLSE when the loss and gain exactly cancel each other at any propagation point. Evidently, the NFT technique can stay viable in optical transmission engineering only if the NLSE stays to be an accurate enough model to describe the light evolution in a real optical system. However, in a real-world transmission we always have features that are at odds with the integrable NLSE model, to name the most important two: optical noise and fibre loss [17]. The former is usually studied and incorporated into the integrable model using a perturbation approach [3], [16], resulting in the slightly-perturbed stochastic evolution of modulated NFT spectrum. The latter can be dealt with in two different ways. First, in fibre links using distributed second-order Raman amplification compensating for the fibre loss, the signal power can be assumed to stay almost constant along the propagation [18]-[20], which leads us to an effectively unperturbed NLSE (this case was automatically assumed in the majority of initial NFT works). At the same time, the lumped EDFA schemes are widely deployed in modern fibreoptic communication systems dominating over any other types of amplifiers. When a periodic amplification (e.g., using EDFA) is implemented, it is possible to attribute the integrable NLSE version to the path-average evolution of signal [12], [21]-[23]. In this work we demonstrate how to improve the performance of NFT-based systems using specific properties of the perturbation theory developed for NLSE in the case of EDFA system. We show that the accuracy of the path-average description can be improved through a simple design of the NFT-based links by using the special launching and detecting points of the system leading to an improvement in the $\mathrm{Q}^{2}$-factor which is calculated using the EVM between the received and transmitted QAM symbols.

The paper is organised as follows. First, we recall the basic features of the path-average model. Then we describe (without much details) the NFT-based communication system designs: we consider the NFT communication in the burst mode [11], [12], dealing with the so-called nonlinear inverse synthesis 
technique [7], [11], [12], and after that describe the NFT communication system that uses periodically-continued signals and operates with the periodic version of NFT processing [24]-[26]. Finally, we present and discuss the results of our numerical modelling and the system performance. Our findings are summarised in the Conclusion.

\section{Master Model fOR the Optical Signal TRANSmission IN FIBRE}

The integrable NLSE for some field function $q$ in a normalised form (see the details of normalisation for the optical-fibre systems in e.g., [4], [21], [23]) reads

$$
i \frac{\partial q}{\partial z}+\frac{1}{2} \frac{\partial^{2} q}{\partial t^{2}}+|q|^{2} q=0
$$

where in the case considered in our paper, i.e., for the light propagation down the fibre, $t$ is the retarded time variable in the frame moving with the group velocity of the light envelope and $z$ is the distance along the fibre. (1) is integrable in the sense that it can be exactly solved through using the NFT formalism [1] (the "inverse scattering method" name is widely used in mathematical literature) in a manner that is very similar to the use of conventional Fourier transform applied for the solution of linear partial differential equations (see [3], [4] and works cited therein).

However, in fact, the lossless NLSE is an approximation of the Maxwell equations describing the propagation of an electromagnetic field in the fibre. Considering only the second order dispersion and taking into account the fibre loss, Kerr nonlinearity, and periodic amplification, the perturbed NLSE (in the normalised form, again) becomes [23]:

$$
\begin{aligned}
i \frac{\partial q}{\partial z}+\frac{1}{2} \frac{\partial^{2} q}{\partial t^{2}}+|q|^{2} q= & -i \Gamma q+i\left(e^{\Gamma L}-1\right) \\
& \times \sum_{n=1}^{N} \delta(z-n L) q,
\end{aligned}
$$

where $q$ is the envelope of the field, $L$ is the normalized span length, $N$ is the number of amplifiers and $\Gamma$ is the fibre loss coefficient. Equation (2) is not integrable, i.e., its exact solution cannot be explicitly presented as the simple evolution of NFT modes. However, a simple change of variable $q(z, t)=a(z) Q(z, t)$, where $a(z)$ satisfies the relation

$$
\frac{d a}{d z}=-\Gamma a+\left(e^{\Gamma L}-1\right) \times \sum_{n=1}^{N} \delta(z-n L) a,
$$

leads to the following equation for the new function $Q$ :

$$
i \frac{\partial Q}{\partial z}+\frac{1}{2} \frac{\partial^{2} Q}{\partial t^{2}}+a(z)^{2}|Q|^{2} Q=0,
$$

which is the NLSE with the $z$-dependent nonlinear parameter. Following [21], we decompose the signal $Q$ and nonlinear varying parameter $a$ in the form of a sum of average and oscillating (marked by tildes) parts: $Q(z, t)=Q_{0}(z, t)+\tilde{Q}(z, t)$ and $a(z)^{2}=1+\tilde{a}(z)$ with $\langle\tilde{Q}(z)\rangle=\langle\tilde{a}(z)\rangle=0$ where the average $\langle\cdot\rangle$ is taken over one span of the length $L$. Then, the average function $Q_{0}(z, t)$ obeys the following equation:

$$
\begin{aligned}
& i \frac{\partial Q_{0}}{\partial z}+\frac{1}{2} \frac{\partial^{2} Q_{0}}{\partial t^{2}}+\left|Q_{0}\right|^{2} Q_{0} \\
& \quad+[\text { higher order terms involving } \tilde{Q}(z)]=0,
\end{aligned}
$$

where

$$
\tilde{Q}(z)=i \tilde{a}_{1}(z)\left|Q_{0}\right|^{2} Q_{0}
$$

up to the order of corrections $\sim L^{2}$ (see the Appendix for more details), and $\tilde{a}_{1}(z)$ is explicitly given by the expression

$$
\tilde{a}_{1}(z)=\frac{1}{2 \Gamma}+\frac{L}{2}-z-\frac{L e^{-2 \Gamma z}}{1-e^{-2 \Gamma L}}, \quad 0<z<L .
$$

This means that the solution to (4) can be presented as a combination of the contribution that is governed by the (lossless) NLSE, (5), up to order $O(L)$, and a component describing deviations of the full solution of (2) from the solution of pure NLSE due to the periodic variations of loss and gain [21]:

$$
q(z, t)=\Lambda e^{-\Gamma z^{\prime}}\left[Q_{0}+\tilde{Q}\left(z^{\prime}\right)\right],
$$

where $z=n L+z^{\prime}, 0<z^{\prime}<L$, and

$$
\Lambda^{2}=\frac{2 \Gamma L}{1-e^{-2 \Gamma L}} .
$$

In the next section we explain how to exploit these expressions to improve the performance of fibre-optic communication systems based on NFT.

\section{NFT-BASED COMMUNICATION SySTEMS}

To avoid unnecessary repetitions, we refer readers for the details of the NFT operations to the recent review paper [4]. In simple terms, it is possible to present the evolution of any decaying signal governed by (1) by the equivalent evolution of nonlinear spectral modes defined inside the NFT spectrum domain in a unique manner, where evolution of each nonlinear spectral component along the fibre becomes trivial, decoupled, and linear. Taking advantage of this property, it is possible to map our data on the NFT spectrum, generate the corresponding profile in the space-time domain and then launch the resulting signal into the fibre. At the receiver, the data-carrying NFT spectrum is extracted from the (usually distorted) signal and after a simple equalisation (to reverse the trivial evolution of NFT spectrum), the transmitted data is retrieved. In the context of the distortions produced by the periodic alternation of loss and gain, given the "approximately integrable" NLSE (5) one can construct $Q_{0}$ for which the nonlinear spectrum contains the data to be transmitted. The resulting $Q_{0}$ is the path-averaged of the solution of the actual NLSE describing the lossy fibre, (2). Next, from $Q_{0}$ we construct the function $q(0, t)$ according to the rule given by (8) ignoring the higher order terms and launch it into the lossy system where the light evolution is governed by (2).

$Q_{0}$ is only the average of the actual signal travelling through the fibre over one span length. The presence in (5) of the additional term $\tilde{Q},(6)$, results in the deviation of the solution for the (almost) integrable NLSE, (5), from the actual solution for 


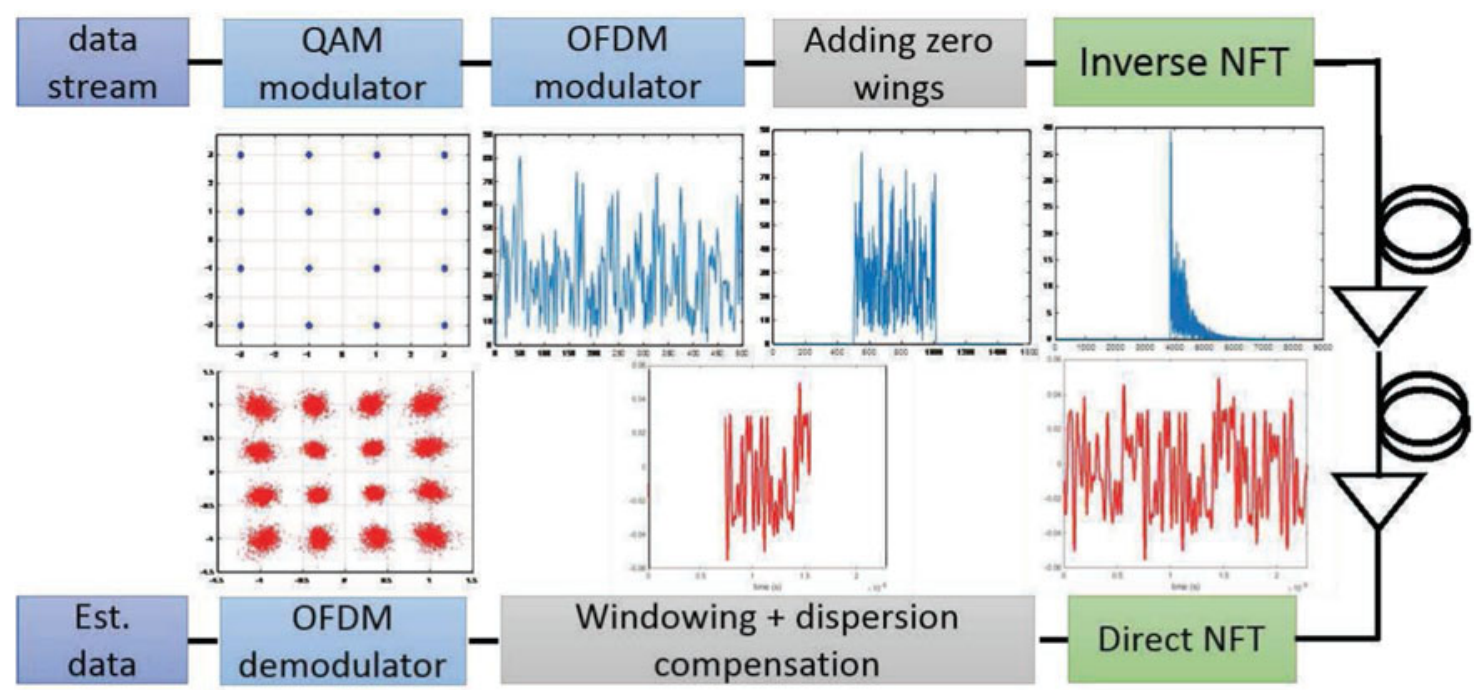

Fig. 1. An NIS system. At the transmitter the signal whose continuous NFT spectrum matches the linear spectrum of an encoded (here an OFDM) signal is generated. At the receiver, the continuous spectrum is caltabculated and the encoded data (corrupted by noise) is retrieved.

the complete model (2). This additional term entails two kinds of distortion/error in such a system. The first type comes from the higher order terms of the fluctuating part $\tilde{Q}$ omitted in (5). The second source of error emerges from our constructing the function $q$ from the given $Q_{0}$ according to (8), i.e., from the presence of the oscillatory $\tilde{Q}$ in (8). The latter source of error takes its minimum value at two spatial points, $z_{1,2}$, where the condition $\tilde{a}_{1}(z)=0$ is satisfied, according to definition (6). At such points in $z$, the average of the solution to (2) is truly a solution to (5) up to order $O(L)$, though there is still an accumulated error ensuing from the higher orders terms disregarded in (5). Therefore, launching and detecting our signal at these spatial points $z_{1,2}$ reduces the overall error. This approach is known in the soliton communication systems [27]-[30], and in this paper we apply it to the NFT transmission. We would like to stress that this technique can be applied to any NFT-based communication system (or to any system relying in its design and functionality on the properties of the integrable NLSE model), so the particular NFT-based systems considered below provide just examples of the technique utilisation.

In what follows, we evaluate improvement in the performance of two different NFT-based communication systems resulting from the use of the technique described above. The first example is the nonlinear inverse synthesis (NIS) system, introduced in [7] and assessed in [11], [12], in which the data is mapped on the continuous NFT spectrum of a signal having a finite extent (i.e., corresponding to NFT for decaying signals). The experimental evaluations of such a system has been taken place in [31]-[33] showing the potential of NFT-based systems in the mitigation of nonlinear transmission impairments. The second example is a system based on the periodic NFT, introduced in [34] and studied in detail in [25], [26], in which the data is mapped on the main spectrum of the periodically continued signal. As the periodic NFT spectrum is discrete, the latter situation is different from the NIS method, and the similarity in the improvement of performance for both cases demonstrates the generality of the path-average approach.

\section{A. Nonlinear Inverse Synthesis NFT Systems}

The diagram representing the sequence of operations for the NIS scheme is shown in Fig. 1: here the conventional data is mapped onto the continuous part of the NFT spectrum of the signal. This is done numerically through starting out from a given (encoded) continuous NFT and then performing the inverse NFT (INFT) operation [35] to recover the corresponding profile in time domain. Here, without loss of generality and for the illustration purpose, we use the spectrum of an ordinary orthogonal frequency-division multiplexing (OFDM) signal as a starting point to get the profile in space-time domain $Q_{0}(0, t)$. It means that we construct a solution of (5), ignoring the higher order terms, whose continuous NFT spectrum matches the spectrum of OFDM signal. (As shown in [11], OFDM is the better option for NIS due to its good spatial localization). Then the obtained $Q_{0}$ is used to get $q(0, t)$ through (8), ignoring the higher order terms and the resulting signal is sent to the system with periodically implanted amplifiers (see Fig. 2). After receiving the signal, the pre-factor $\Lambda e^{-\Gamma z^{\prime}}$ in (8) is removed. Then we compute the NFT spectrum of the resulting waveform, and finally the data-carrying OFDM signal (or rather its spectrum) is retrieved by applying a one step equalisation, as the NLSE channel plays the role of the all-pass filter for the continuous NFT spectrum part [11], [12]. In our simulations, the fibre parameters have the typical values: the dispersion coefficient is $\beta_{2}=-20 \mathrm{ps}^{2} / \mathrm{km}$, the Kerr nonlinearity coefficient is $\gamma=1.22 / \mathrm{W} / \mathrm{km}$, and the attenuation coefficient is $\alpha=0.2 \mathrm{~dB} / \mathrm{km}$. The link consists of several spans of lossy fibre with an amplifier at the end of each span compensating for the span loss. Thus, the first amplifier overshoots the signal above its power loss during the first part of the link. The OFDM signal is constructed with 256 sub-carriers 


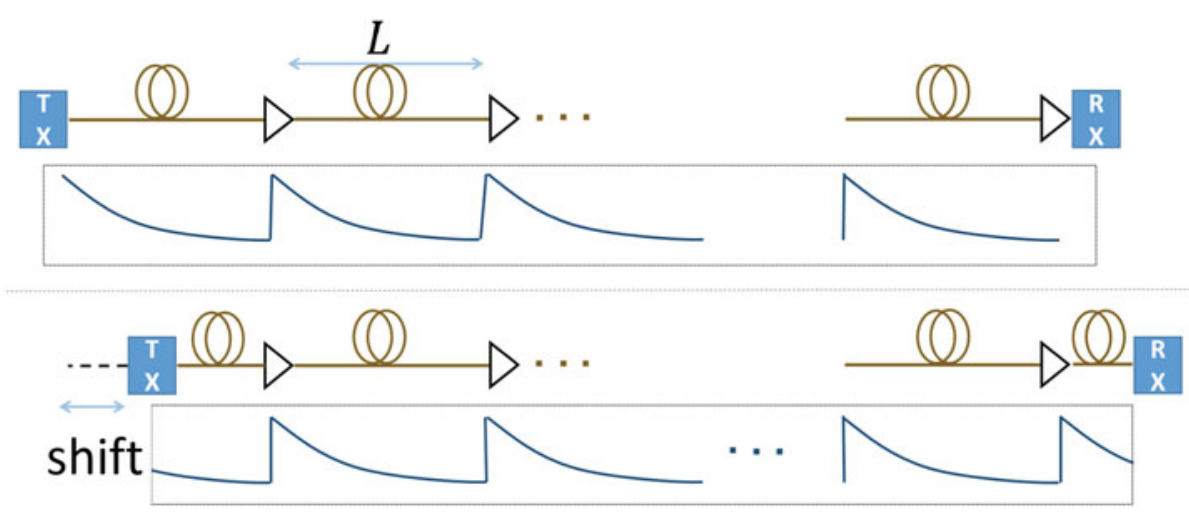

Fig. 2. Schematic of the optical link with lumped amplifier and the power profile along it for a conventional set up (top scheme), and the optimised system where the amplifiers are shifted to reduce the accumulated error of the path-average model (bottom scheme).

each carrying a QPSK symbol forming a 3 ns signal amounting to the overall bandwidth of $85 \mathrm{GHz}$.

\section{B. Periodic NFT Systems}

Periodic NFT (PNFT) can be advantageous to other NFTbased designs in terms of the simpler encoding scheme and reduced processing load at the receiver [24]. Since in the inverse stage of designing the encoded signal at the transmitter the PNFT does not require any zero wings, the consecutive symbols can be transmitted continuously (i.e., without the "gaps" in the stream otherwise required by the methods employing ordinary NFT) leading to a higher spectral efficiency [26]. PNFT communication system proposed in [25] is based on mapping the encoded data onto the discrete points of the invariant main spectrum of the signal; a similar block diagram as the one shown in Fig. 1 without the OFDM modulator/demodulator and inserting cyclic prefix instead of zero wings delineates the system, for more details and definitions see [24], [25]. In our system we use the simple approximation of perturbed plane wave [24], [36]. A plane wave basically acquires a single (nondegenerate) point in its main spectrum and a few double (degenerate) ones which can further split up into pairs of single points. These single points are the ones determining the behaviour of the signal. Following the steps explained in [25, Sec. 3.2], we can control the aperture between points of each pair allowing to modulate it according to the data to transmit [25], [36]. Here, we simulate a periodic NFT based communication system with a 16-QAM constellation and $1 \mathrm{GSym} / \mathrm{s}$. Symbols are separated using cyclic extensions according to the distance to which the signal is sent, with the duration of each extension slightly exceeding the dispersion-induced memory $\Delta T_{\text {ext }}=2 \pi \beta_{2} W Z$, where $W$ is the signal bandwidth and $Z$ is the overall transmission distance.

\section{Simulation Results}

Firstly, we address the case of NIS-based transmission, Section III-A. At the beginning, we demonstrate that the main contribution to the improvement in $\mathrm{Q}^{2}$-factor stems from our diminishing the error between the real system and its approximation by the NLSE model. This can be made evident by showing the correlation between the overall $\mathrm{Q}^{2}$-factor and the

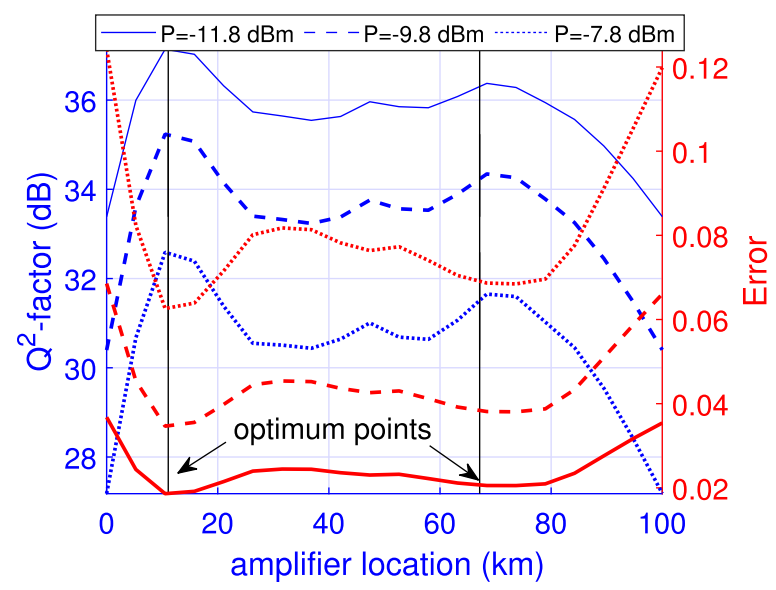

Fig. 3. $\mathrm{Q}^{2}$-factor and error (10) against location of the amplifier showing a correlation between the two for a link with 10 spans of length $L_{\text {span }}=100 \mathrm{~km}$ and signal power of $-11.8,-9.8$ and $-7.8 \mathrm{dBm}$. The analytically obtained optimum launch and/or detection points are marked by a vertical line.

error between the data obtained at the receiver using the processing of the signal constructed by means of (5)-(8) and by using the true signal obtained from the solution of the full (2). In Fig. 3 we depict the error and the $\mathrm{Q}^{2}$-factor against the shift in the amplifier location showing the correlation between the two in a link consisting of 10 spans of length $L_{\mathrm{span}}=100 \mathrm{~km}$. In this figure, keeping the length of the link and number of amplifiers fixed, all amplifiers are shifted with the value shown in the $\mathrm{x}$-axis. Hereafter, the signal power is defined as the power of the signal at the end of the first amplifier. The error in Fig. 3 is defined as the $l_{1}$-norm of the difference between the solution of (2), $q(Z, t)$, and $\Lambda e^{-\Gamma z^{\prime}} Q_{0}(Z, t)$ at the end of the link having the total length $Z$ :

$$
e=\frac{\int_{t}\left|\Lambda e^{-\Gamma z^{\prime}} Q_{0}(Z, t)-q(Z, t)\right| d t}{\int_{t}|q(Z, t)| d t},
$$

where $z^{\prime}$ is the location of the first amplifier. Now we turn to studying the improvement in the performance resulting from shifting the location of the amplifiers to the optimum points, $z_{1}$ or $z_{2}$, at which $\tilde{a}_{1}$ defined by (7) is exactly zero. Fig. 4(a) shows the result for the noiseless system consisting of 10 spans 


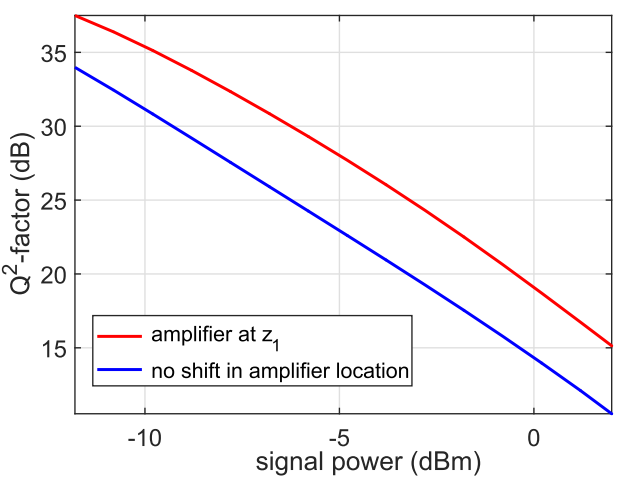

(a)

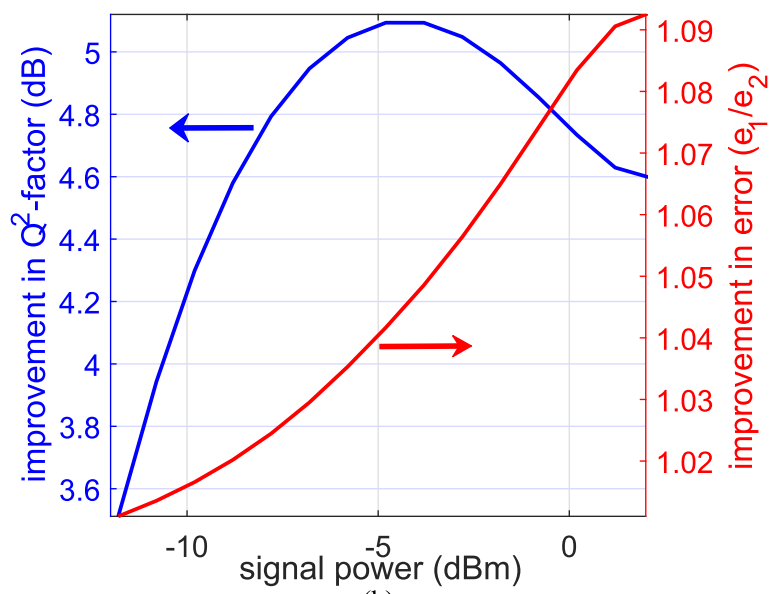

(b)

Fig. 4. (a) $\mathrm{Q}^{2}$-factor vs signal power with/without amplifier shift, (b) improvement in $\mathrm{Q}^{2}$-factor and error $\left(e_{1}\right.$ and $e_{2}$ of (10) for the case w/o and w/ shifting the amplifier location respectively) by shifting the amplifier without noise where the link consists of 10 spans of length $L_{\text {span }}=100 \mathrm{~km}$ for the OFDM-NIS system of Section III-A.

of length $100 \mathrm{~km}$, so the total system length is $1000 \mathrm{~km}$. This figure compares the $\mathrm{Q}^{2}$-factor behaviour for two cases: for the system with zero shift in the amplifier location and for the system where we shifted the amplifier location to the point $z_{1}$. From this figure we see that we can gain a minimum improvement of $5 \mathrm{~dB}$ when the location of the amplifiers is optimised.

As it is shown in [27], in a split-step Fourier analysis of the signal evolution, the commutator, $[\hat{D}, \hat{N}]$, between the linear and nonlinear operators

$$
\hat{D}=-\frac{i}{2} \frac{\partial^{2}}{\partial t^{2}}, \quad \hat{N}=-i|q|^{2}
$$

contributes to the error risen from the path-average model in a form of a prefactor to $\tilde{a}_{1}(z)$. Hence, the actual error coming from the approximations in (5)-(8) also depends on the value of the commutator, and the latter increases with the growing signal power. Therefore, one might expect a higher improvement in the performance ensuing from our shifting the amplifier location when we operate with signals having higher powers. However, as shown in Fig. 4(b) this tendency is true at the beginning but after some threshold power value the optimised system performance starts to drop down. This discrepancy is explained by the fact that the overall performance of the NIS system also massively depends on the errors induced by the numerical NFT

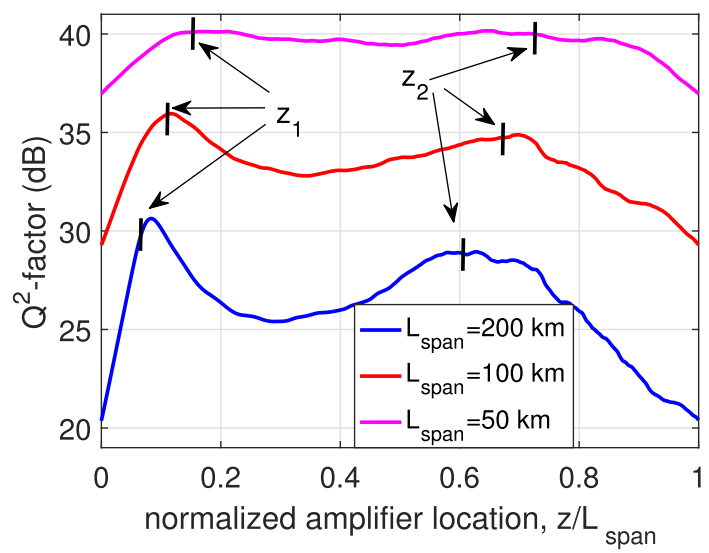

Fig. 5. $Q^{2}$-factor vs normalised amplifier location for different lengths of span in a noiseless scenario for a signal with power $P=-8.8 \mathrm{dBm}$ for the OFDMNIS system described in Section III-A in a $1000 \mathrm{~km}$ link. The analytically obtained optimum launch and/or detection points are marked by a vertical dash for each $L_{\text {span }}$.

routines: these errors become significant at high signal powers [11] and, in fact, start to be the determining factor in the performance degradation of NIS system. Hence, as it is seen from Fig. 4(b), while the improvement of the agreement between the approximate and true models increases with power, the gain in the $\mathrm{Q}^{2}$-factor reaches a maximum and then starts to decay when we enter the high powers region. This decline in the performance at high powers can only be rectified by increasing the number of samples. Specially, to have an acceptable level of accuracy in the inverse NFT stage, the time window in which the signal is defined, should be large enough to contain as much of the signal energy in the decaying wings as possible. This in turn leads to a significant computational complexity beyond the capacity of the computers we use to simulate the system.

The impact of the span length is also visible from Fig. 5, in which the $\mathrm{Q}^{2}$-factor is plotted against the normalized location of amplifiers for $L_{\text {span }}=50,100,200 \mathrm{~km}$. This figure reveals the compliance of the theoretically predicted optimum points $z_{1,2}$ with the optimised locations (corresponding to the maxima in $Q^{2}$-factor) obtained in the simulation. This figure also demonstrates the overall increase of the error as the result of the growing span length.

The simulations described above have been so far performed without taking noise into account, just demonstrating the performance improvement with the optimisation of amplifier locations. Now we consider how the optimisation technique works in the presence of the ASE noise. Fig. 6 shows the effectiveness of using the optimal launch and detection points and a noticeable improvement in the $\mathrm{Q}^{2}$-factor behaviour (as a function of power) for a link consisting of 10 spans of length $100 \mathrm{~km}$ with loss, when the additive ASE noise is added at the end of each span. To obtain this results, $2^{16}$ 16QAM symbols are transmitted. The dependency of the performance on the signal power and bandwidth is illustrated in Fig. 9 in which the error between the real model and the path-averaged one (this error is again defined by (10)) and the $\mathrm{Q}^{2}$-factor for the two cases are shown. As can be seen from this figure, an improvement in the performance is obtained at all powers and bandwidth values. An almost linear 


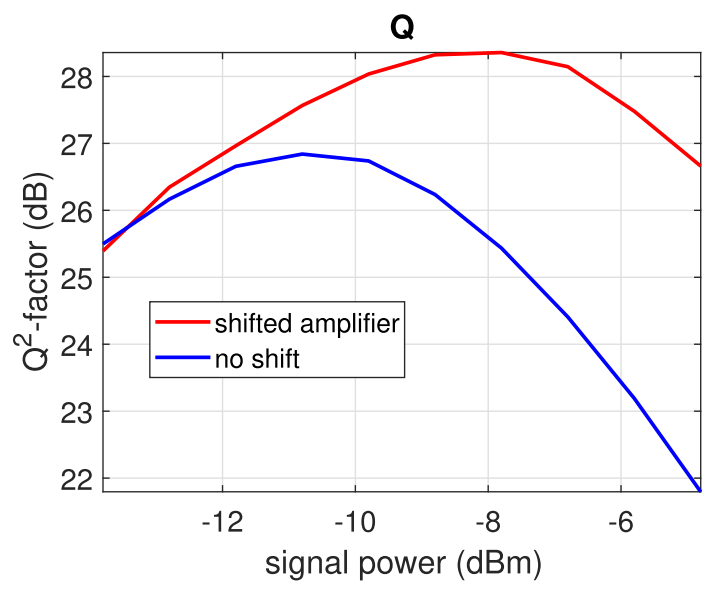

Fig. 6. $\mathrm{Q}^{2}$-factor vs signal power with/without amplifier shift in a link of 10 spans of length $L_{\text {span }}=100 \mathrm{~km}$ for the OFDM-NIS system with noise.

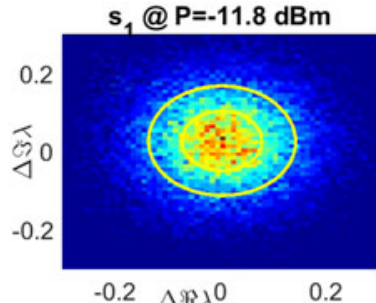

(a)

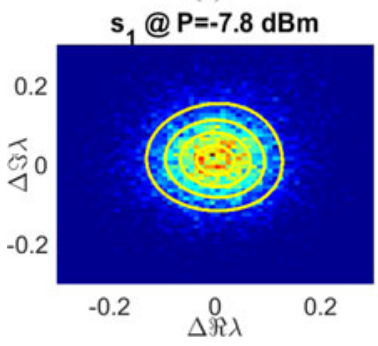

(c)

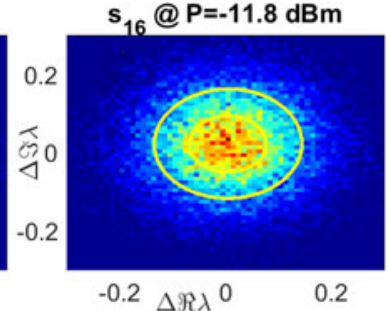

(b)

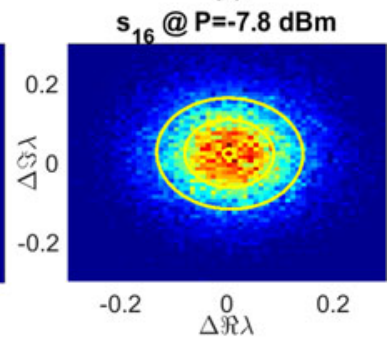

(d)
Fig. 7. 2D histogram of the error in the received constellation and the fitted Gaussian distribution for (a) symbol $s_{1}$ close to the origin of a 16QAM constellation at power $P=-11.8 \mathrm{dBm}$, (b) symbol $s_{16}$ away from the origin of a 16QAM constellation at power $P=-11.8 \mathrm{dBm}$, (c) symbol $s_{1}$ close to the origin of a 16QAM constellation at power $P=-7.8 \mathrm{dBm}$ and (d) symbol $s_{16}$ close to the origin of a 16QAM constellation at power $P=-7.8 \mathrm{dBm} . \Delta \Im \lambda$ and $\Delta \Re \lambda$ represent the imaginary and real part of the displacement from the transmitted constellation point respectively.

improvement in the performance with bandwidth (larger than $40 \mathrm{GHz}$ ) can be inferred from Fig. 9. This figure yet again makes it evident that the main contributor to the performance is the error between the two models.

It is worth mentioning that we use $\mathrm{Q}^{2}$-factor as a measure of system performance as is conventionally used in the fibreoptic communication community. Using this figure implies the assumption of Gaussianity for the received points in the constellation which in an NFT-based system is not a trivial fact. However, as is shown in Fig. 7 through a 2D histogram of the received points, although with signal dependent parameters, noise can be considered as a Gaussian random variable. Fig. 7 attributes to the received points of an OFDM-NIS system with 256 subcarriers at the end of a $1000 \mathrm{~km}$ link and shows the

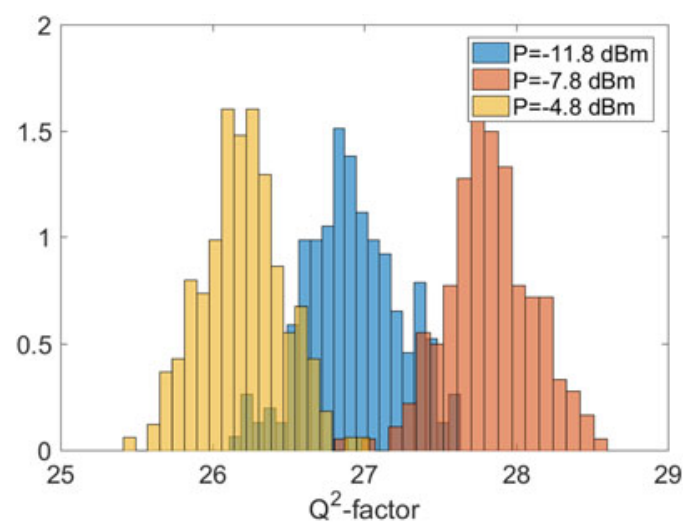

Fig. 8. The histogram of the calculated $\mathrm{Q}^{2}$-factor of three points of Fig. 6 at powers $P=-11.8,-7.8,-4.8 \mathrm{dBm}$ at the end of a $1000 \mathrm{~km}$ link where 200 measurements of $\mathrm{Q}^{2}$-factor each obtained from $2^{9}$ 16QAM symbols are used.

histogram of the displacement of the received complex points in the nonlinear Fourier domain from the transmitted ones for two sets of constellation points; close to the centre $\left(s_{1}\right)$ and far away from it $\left(s_{16}\right)$ for two values of power $P=-11.8 \mathrm{dBm}$ and $P=-7.8 \mathrm{dBm}$. As the power increases the distribution deviates from Gaussian. To obtain these histograms, $2^{16}$ 16QAM symbols are used. For the PNFT symbols, similar results are reported in [37] confirming that the received constellation points in the nonlinear Fourier domain acquire a Gaussian distribution to some level of approximation.

To confirm the validity of results from the Monte Carlo simulation, we also present in Fig. 8 the histogram of the $\mathrm{Q}^{2}$-factor for three points of Fig. 6 at $P=-11.8,-9.8,-7.8 \mathrm{dBm}$. For each calculation of the $\mathrm{Q}^{2}$-factor, $2^{9} 16 \mathrm{QAM}$ symbol are used and the averaging is done over 200 measurement. This figure clearly shows the reliability of the measurements for the standard deviation is small in all three cases.

For the PNFT system from Section III-B, the same simulations have been performed basically showing similar tendency in the system performance when the amplifier locations are optimised using the theory from Section II. Fig. 10 compares the $\mathrm{Q}^{2}$-factor of the system against the amplifier location for three lengths of span for a signal with a relatively high power of $0 \mathrm{dBm}$ in a noiseless link. As is expected for such a high power, the difference between the performance for systems with the amplifiers located at the optimum point and for those with the amplifiers positioned at the beginning of the fibre is considerable. The next simulation run deals with the situation when we add ASE noise after each span: Fig. 11, again, compares the performance of systems with and without an optimum shift in the location of amplifiers against the transmission distance. This figure clearly demonstrates a considerable advantage for the system with optimum shift of amplifiers. By increasing the length of the link, the accumulated error caused by the mismatch between the two models grows which intensifies the impact of choosing an optimum launch/detection point. In Fig. 11 we also depicted the transmitter and receiver constellation for the two cases (at distance $Z=1400 \mathrm{~km}$ ). From this figure we observe that the deterministic error for the PNFT system (appearing in the form of a shift in the received points) is clearly more 


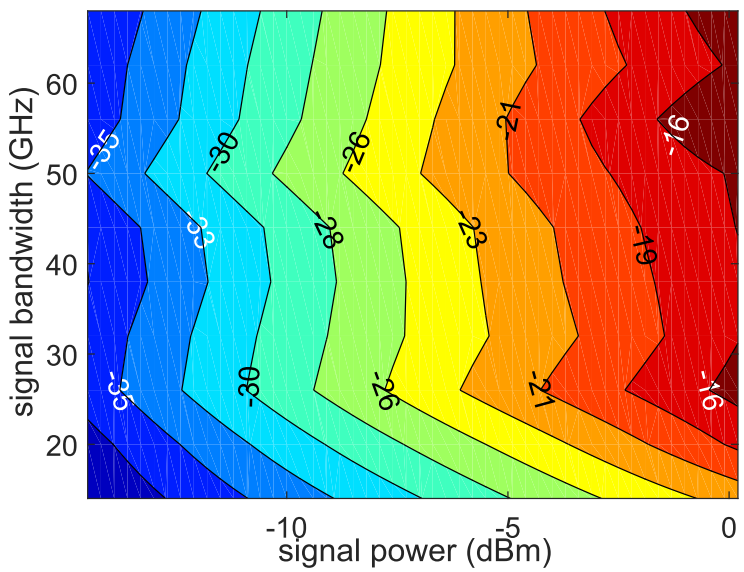

(a)

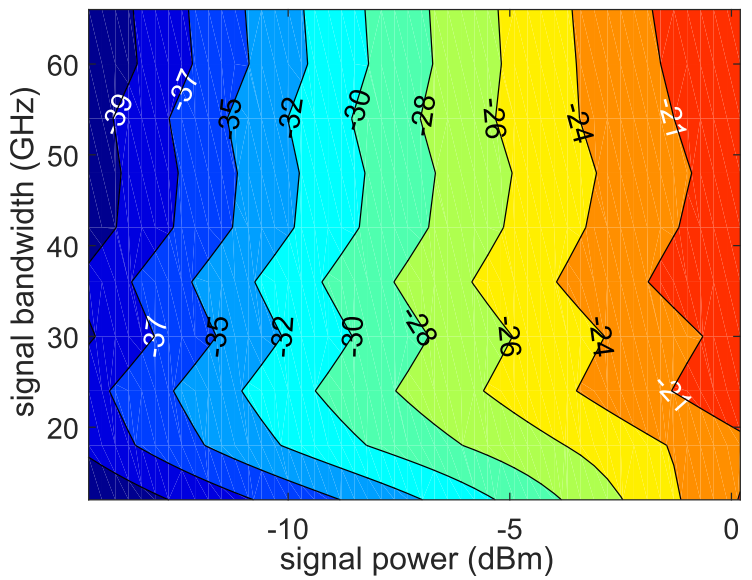

(c)

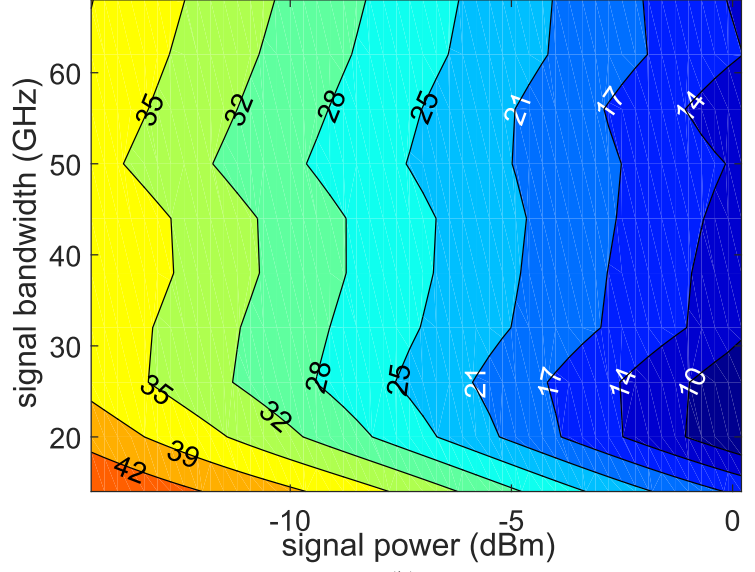

(b)

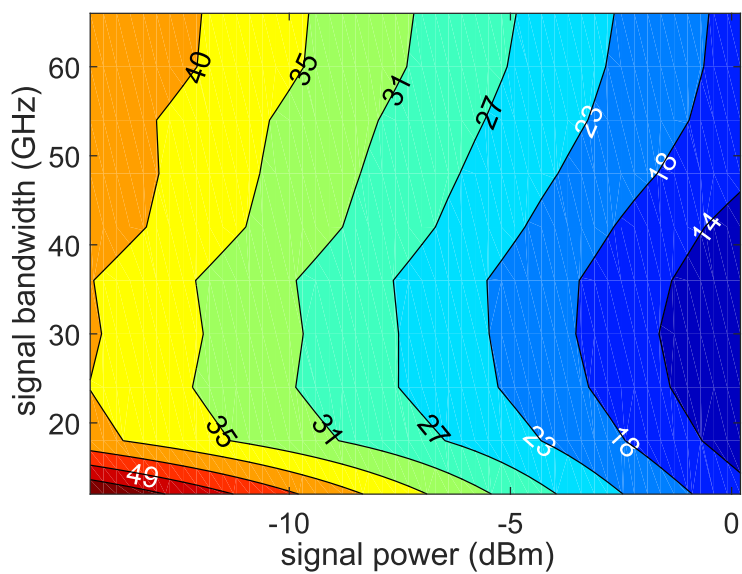

(d)

Fig. 9. (a) Error and (b) $\mathrm{Q}^{2}$-factor (in $\mathrm{dB}$ ) for the case without shift in the amplifier location, and (c) error and (d) $\mathrm{Q}^{2}$-factor (in $\mathrm{dB}$ ) with optimum amplifier location. For all panels: 10 spans of length $L_{\text {span }}=100 \mathrm{~km}$, the system is the OFDM-NIS described in Section III-A.

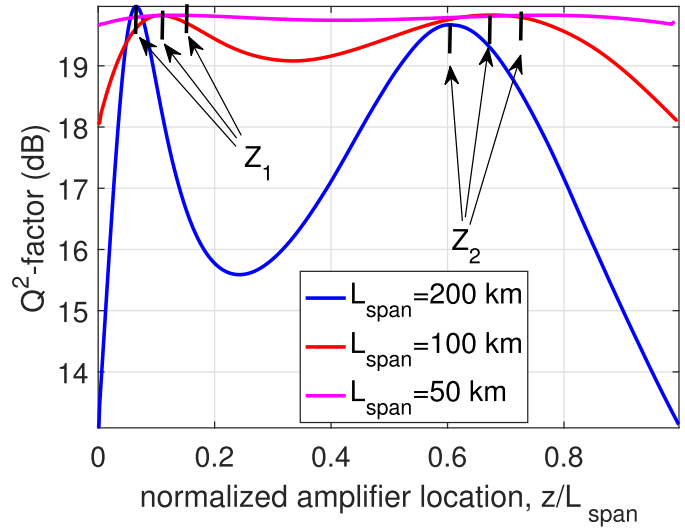

Fig. 10. $\mathrm{Q}^{2}$-factor vs normalised amplifier location for different lengths of span in a $1000 \mathrm{~km}$ link with PNFT in a noiseless scenario for a signal with power $P=0 \mathrm{dBm}$ for the system described in Section III-B.

pronounced when the amplifier location is not optimised. Since the error incurred from the inaccuracy of the path-averaged model is deterministic, one can reduce its impact by de-skewing the received constellation using some pilots (transmitted symbols known to the receiver). While introducing such techniques improves the performance (at the expense of losing data rate),

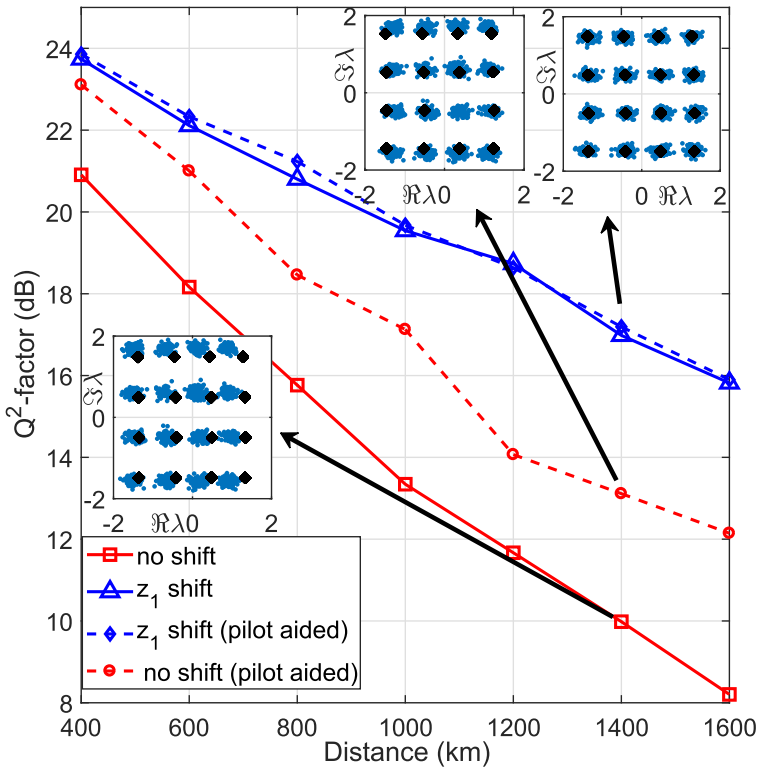

Fig. 11. $\mathrm{Q}^{2}$-factor vs distance of a 16QAM constellation for the two cases of no shift and $z_{1}$ shift in amplifier location when signal power is $P=0 \mathrm{dBm}$ and $L_{\text {span }}=200 \mathrm{~km}$ in PNFT-based system of Section III-B. In the insets, the transmitted (black) and received (light blue) constellation for two cases are shown at the distance $z=1400 \mathrm{~km}$. 
the improvement is limited as is depicted in Fig. 11. In obtaining these result a pilot as long as $2^{7} 16 \mathrm{QAM}$ symbols is used until the performance improvement is considerable.

\section{CONCLUSION}

In this work, we applied an approach known from the theory of soliton communications to improve the performance of the NFT-based transmission systems in fibre links with lumped EDFA amplifiers. The optimisation can be implemented by using special optimal launch and detection points within the first and last spans. In this way, at no extra processing cost, the accumulated error coming from the periodic loss/amplification can be minimised. We implemented this method in two communication systems based on NFT: i) for the NIS system that utilises the continuous spectrum of a vanishing signal, and ii) for the PNFT-based system that uses the main NFT spectrum of a periodic signal. For both types of our systems we demonstrated a clear performance improvement for different span lengths, powers and signal bandwidths, when the location of amplifiers is optimised.

\section{APPENDIX}

In this appendix we derive the path-average model and its higher order terms contributing to the error of modelling in (5)-(8). Separating the operations in (4), let us define

$$
\begin{aligned}
H_{0}\left(Q, Q^{*}\right) & =-\frac{1}{2} \frac{\partial^{2} Q}{\partial t^{2}}-|Q|^{2} Q, \\
\text { and } \quad \bar{H}_{0}\left(Q, Q^{*}\right) & =-|Q|^{2} Q,
\end{aligned}
$$

from (4) we have

$$
i \frac{d Q}{d z}=H_{0}\left(Q, Q^{*}\right)+\tilde{a}(z) \bar{H}_{0}\left(Q, Q^{*}\right) .
$$

By a change of variable to represent $Q$ in terms of infinitesimal deviations from the average $Q_{0}$, one can separate the average term from higher order ones in (13) as below

$$
\begin{aligned}
i \frac{\partial Q_{0}}{\partial z}= & -\frac{1}{2} \frac{\partial^{2} Q_{0}}{\partial t^{2}}-\left|Q_{0}\right|^{2} Q_{0} \\
& +\frac{1}{2}\left\langle\tilde{a} \tilde{a}_{2}\right\rangle\left[\bar{H}_{0},\left[\bar{H}_{0}, H_{0}\right]\right]+O\left(L^{3}\right),
\end{aligned}
$$

with

$$
\frac{d \tilde{a}_{2}}{d z}=\tilde{a}_{1}, \quad\left\langle\tilde{a}_{2}\right\rangle=0,
$$

where $\left[\bar{H}_{0}, H_{0}\right]$ is the Lie bracket (see [38] for more details):

$$
\left[\bar{H}_{0}, H_{0}\right]=-\left(2 Q_{0}\left|Q_{0}\right|^{2}+Q_{0}^{*} Q_{0 t}^{2}+Q_{0}^{2} Q_{0}{ }_{t t}^{*}\right),
$$

where the subscript represents the time derivative. In this way, $Q$ is related to $Q_{0}$, up to $O\left(L^{2}\right)$, through the following expression:

$$
\begin{aligned}
Q= & Q_{0}+i \tilde{a}_{1}\left|Q_{0}\right|^{2} Q_{0}-\frac{1}{2} \tilde{a}_{1}^{2}\left|Q_{0}\right|^{4} Q_{0} \\
& +\tilde{a}_{2}\left[\bar{H}_{0}, H_{0}\right]+O\left(L^{3}\right) .
\end{aligned}
$$

Since $\tilde{a}_{1}^{2}$ and $\tilde{a}_{2}$ are of order $O\left(L^{2}\right)$, keeping the corrections to $Q_{0}$ up to the first order gives (5) and (6).

\section{REFERENCES}

[1] V. E. Zakharov and A. B. Shabat, "Exact theory of two-dimensional selffocusing and one-dimensional self-modulation of waves in nonlinear media," Soviet Phys.-JETP, vol. 34, pp. 62-69, 1972.

[2] A. Hasegawa and T. Nyu, "Eigenvalue communication," J. Lightw. Technol., vol. 11, no. 3, pp. 395-399, Mar. 1993.

[3] M. I. Yousefi and F. R. Kschischang, "Information transmission using the nonlinear Fourier transform, Parts I-III," IEEE Trans. Inf. Theory, vol. 60, no. 7, pp. 4312-4369, Jul. 2014.

[4] S. Turitsyn, et al."Nonlinear Fourier transform for optical data processing and transmission: Advances and perspectives," Optica, vol. 4, pp. 307322, 2017.

[5] E. G. Turitsyna and S. K. Turitsyn, "Digital signal processing based on inverse scattering transform," Opt. Lett., vol. 38, pp. 4186-4188, 2013.

[6] J. Prilepsky, S. A. Derevyanko, and S. K. Turitsyn, "Nonlinear spectral management: Linearization of the lossless fiber channel," Opt. Express, vol. 21, pp. 24344-24367, 2013.

[7] J. E. Prilepsky, S. A. Derevyanko, K. J. Blow, I. Gabitov, and S. K. Turitsyn, "Nonlinear inverse synthesis and eigenvalue division multiplexing in optical fiber channels," Phys. Rev. Lett., vol. 113, 2014, Art. no. 13901.

[8] S. Wahls and H. V. Poor, "Fast numerical nonlinear Fourier transforms," IEEE Trans. Inf. Theory, vol. 61, no. 2, pp. 6957-6974, Dec. 2015.

[9] S. Wahls, S. T. Le, J. E. Prilepsky, H. V. Poor, and S. K. Turitsyn, "Digital backpropagation in the nonlinear Fourier domain," in Proc. IEEE Int. Workshop Signal Process. Adv. Wireless Commun., Stockholm, Jun. 2015, pp. 445-449.

[10] H. Bülow, "Experimental demonstration of optical signal detection using nonlinear fourier transform," J. Lightw. Technol., vol. 33, no. 7, pp. 14331439, Apr. 2015

[11] S. T. Le, J. E. Prilepsky, and S. K. Turitsyn, "Nonlinear inverse synthesis for high spectral efficiency transmission in optical fibers," Opt. Express, vol. 22, pp. 26720-26741, 2014

[12] S. T. Le, J. E. Prilepsky, and S. K. Turitsyn, "Nonlinear inverse synthesis technique for optical links with lumped amplification," Opt. Express, vol. 23, pp. 8317-8328, 2015.

[13] S. Hari, M. I. Yousefi, and F. R. Kschischang, "Multieigenvalue communication," J. Lightw. Technol., vol. 34, no. 13, pp. 3110-3117, Jul. 2016.

[14] V. Aref, S. T. Le, and H. Buelow, "Demonstration of fully nonlinear spectrum modulated system in the highly nonlinear optical transmission regime," in Proc. Eur. Conf. Opt. Commun., 2016, Paper Th.3.B.2.

[15] S. Hari and F. R. Kschischang, "Bi-directional algorithm for computing discrete spectral amplitudes in the NFT," J. Lightw. Technol., vol. 34, no. 15, pp. 3529-3537, Aug. 2016.

[16] S. A. Derevyanko, J. E. Prilepsky, and S. K. Turitsyn, "Capacity estimates for optical transmission based on the nonlinear Fourier transform," Nature Commun., vol. 7, 2016, Art. no. 12710.

[17] R.-J. Essiambre, G. Kramer, P.J. Winzer, G.J. Foschini, and B. Goebel, "Capacity limits of optical fiber networks," J. Lightw. Technol., vol. 28, no. 4, pp. 662-701, Feb. 2010.

[18] J. D. Ania-Castañón et al., "Ultralong Raman fibre lasers as virtually lossless optical media," Phys. Rev. Lett., vol. 96, 2006, Art. no. 023902.

[19] J. D. Ania-Castañón, V. Karalekas, P. Harper, and S. K. Turitsyn, "Simultaneous spatial and spectral transparency in ultralong fiber lasers," Phys. Rev. Lett., vol. 101, 2008, Art. no. 123903.

[20] T J Ellingham, J. D. Ania-Castañón, R. Ibbotson, X. Chen, L. Zhang, and S. K. Turitsyn, "Quasi-lossless optical links for broad-band transmission and data processing," IEEE Photon. Technol. Lett., vol. 18, no. 1, pp. 268 270, Jan. 2006.

[21] A. Hasegawa and Y. Kodama, "Guiding-center soliton in optical fibers," Opt. Lett., vol. 15, pp. 1443-1445, 1990.

[22] K. J. Blow and N. J. Doran, "Average soliton dynamics and the operation of soliton systems with lumped amplifiers," IEEE Photon. Technol. Lett., vol. 3, no. 4, pp. 369-371, Apr. 1991.

[23] L. F. Mollenauer and J.P. Gordon, Solitons in Optical Fibe, New York, NY, USA: Academic, 2006.

[24] M. Kamalian, J. E. Prilepsky, S. T. Le, and S. K. Turitsyn, "Periodic nonlinear Fourier transform for fiber-optic communications, Part I: theory and numerical methods," Opt. Express, vol. 24, pp. 18353-18369, 2016.

[25] M. Kamalian, J. E. Prilepsky, S. T. Le, and S. K. Turitsyn, "Periodic nonlinear Fourier transform for fiber-optic communications, Part II: eigenvalue communication," Opt. Express, vol. 24, pp. 18370-18381, 2016.

[26] M. Kamalian Kopae, J. E. Prilepsky, S. T. Le, and S. K. Turitsyn, "Spectral efficiency estimation in periodic nonlinear Fourier transform based communication systems," in Proc. Opt. Fiber Commun. Conf., 2017, Paper Th2A.54. 
[27] W. Forysiak, N. J. Doran, F. M. Knox, and K. J. Blow, "Average soliton dynamics in strongly perturbed systems," Opt. Commun., vol. 117, pp. 65$70,1995$.

[28] T.-S. Yang, W. L. Kath, and S. K. Turitsyn, "Optimal dispersion maps for wavelength-division-multiplexed soliton transmission," Opt. Lett., vol. 23, pp. 597-599, 1998.

[29] Y. Kodama and A. Maruta, "Optimal design of dispersion management for a soliton-wavelength-division multiplexed system," Opt. Lett., vol. 22 pp. 1692-1694, 1997.

[30] T.-S. Yang, W. L. Kath, and S. K. Turitsyn, "The multiple-scale averaging and dynamics of dispersion-managed optical solitons," J. Eng. Math., vol. 36, pp. 163-184, 1999.

[31] S. T. Le et al., "First experimental demonstration of nonlinear inverse synthesis transmission over transoceanic distances," in Proc. Opt. Fiber Commun. Conf., OSA Tech. Dig., Anaheim, 2016, Paper Tu2A.1.

[32] S. T. Le, I. D. Philips, J. E. Prilepsky, P. Harper, A. D. Ellis, and S. K. Turitsyn, "Demonstration of nonlinear inverse synthesis transmission over transoceanic distances," J. Lightw. Technol., vol. 34, pp. 2459-2466, 2016.

[33] S. T. Le et al., "Achievable information rate of nonlinear inverse synthesis based 16QAM OFDM transmission," in Proc. 42nd Eur. Conf. Opt. Commun., 2016, Paper Th.2.PS2.SC5.
[34] M. Kamalian Kopae, J. E. Prilepsky, S. T. Le, and S. K. Turitsyn, "Optical communication based on the periodic nonlinear Fourier transform signal processing," in Proc. IEEE 6th Int. Conf. Photon., Kuching, 2016, pp. 1-3.

[35] L. L. Frumin, O. V. Belai, E. V. Podivilov, and D. A. Shapiro, "Efficient numerical method for solving the direct Zakharov-Shabat scattering problem," J. Opt. Soc. Amer. B, vol. 32, pp. 290-295, 2015.

[36] M. Kamalian Kopae, J. E. Prilepsky, S. T. Le, and S. K. Turitsyn, "Periodic nonlinear fourier transform based transmissions with high order QAM formats," in Proc. Eur. Conf. Opt. Commun., 2016, pp. 1-3.

[37] M. Kamalian Kopae, J. E. Prilepsky, S. T. Le, and S. K. Turitsyn, "Statistical analysis of a communication system based on the periodic nonlinear Fourier transform," in Proc. Aust. Conf. Opt. Fibre Technol., Sydney, 2016, Paper no. ATh1C. 4.

[38] A. Hasegawa and Y. Kodama, "Guiding-center Soliton," Phys. Rev. Lett., vol. 66, pp. 161-164, 1991

Authors' biographies not available at the time of publication. 